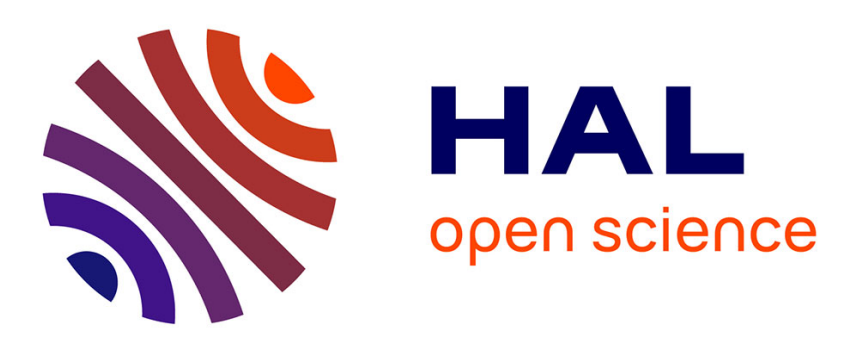

\title{
Parameter Tuning for LV Centralized and Distributed Voltage Control with High PV Production
}

Muhammad A Putratama, Remy Rigo-Mariani, Vincent Debusschere, Yvon Besanger

\section{- To cite this version:}

Muhammad A Putratama, Remy Rigo-Mariani, Vincent Debusschere, Yvon Besanger. Parameter Tuning for LV Centralized and Distributed Voltage Control with High PV Production. IEEE Powertech 2021, Jun 2021, Madrid (virtual), Spain. hal-03293486

\section{HAL Id: hal-03293486 \\ https://hal.science/hal-03293486}

Submitted on 21 Jul 2021

HAL is a multi-disciplinary open access archive for the deposit and dissemination of scientific research documents, whether they are published or not. The documents may come from teaching and research institutions in France or abroad, or from public or private research centers.
L'archive ouverte pluridisciplinaire HAL, est destinée au dépôt et à la diffusion de documents scientifiques de niveau recherche, publiés ou non, émanant des établissements d'enseignement et de recherche français ou étrangers, des laboratoires publics ou privés. 


\title{
Parameter Tuning for LV Centralized and Distributed Voltage Control with High PV Production
}

\author{
Muhammad A. Putratama, Remy Rigo-Mariani, Vincent Debusschere, Yvon Besanger \\ Univ. Grenoble Alpes, CNRS, Grenoble INP ${ }^{\star}$, G2Elab, 38000 Grenoble, France \\ Muhammad-andy.putratama@g2elab.grenoble-inp.fr
}

\begin{abstract}
This paper presents a centralized and a distributed voltage control strategies for a low voltage (LV) grid-connected microgrid with high penetration of photovoltaic (PV) systems. Both strategies are formulated as an optimal power flow (OPF) problem to minimize line losses and PV curtailments. SecondOrder Conic Programming (SOCP) relaxation is formulated to "convexify" the OPF problem. Alternating direction method of multipliers (ADMM) is implemented for the distributed voltage control strategy. Moreover, weighting parameters for both controllers are introduced to have more flexibility in the controller operating mode. A sensitivity analysis on those weighting parameters allows to identify different operating regions, with priority given to loss reduction or curtailment minimization. In particular, we identify an infeasible region in cases where the considered SOCP relaxation is not valid, which highlights the need for fine parameter tuning of the controllers. The effectiveness of both voltage control strategies is validated in a $L V$ radial microgrid.

Index Terms-alternating direction method of multiplier (ADMM), optimal power flow (OPF), photovoltaic (PV) system, second order cone relaxation, voltage control
\end{abstract}

\section{INTRODUCTION}

The number of consumer-owned photovoltaic (PV) systems has been increasing rapidly during the past two decades. Nowadays, a lot of consumers are transforming into "prosumers", referring to users that have a capability to absorb and/or produce energy from/to the utility grid. Moreover, the advancement of technologies and communication infrastructures enable two-way communications between the distribution system operator (DSO) and prosumers (e.g. possibly via a third actor, like an aggregator). These phenomenons enable the transformation of distribution grids into large variety of connected microgrids [1]. The high integration of PV systems in distribution grids uncovers potential benefits for both DSOs and prosumers. It can reduce transmission losses for the utility by producing energy closer to the point of consumption and lower the prosumer's energy bill from self-consumption and by selling their excess production to the grid. However, the intermittent nature of solar radiance may lead to serious voltage fluctuations beyond the accepted limits in cases where the PV installation are not properly sized. If conventional grid assets (e.g., on-load tap changer or capacitor bank) are not enough to ensure the voltage regulation, the PV production must be curtailed which incurs possible economic loss of revenue for

\footnotetext{
${ }^{\star}$ Institute of Engineering Univ. Grenoble Alpes
}

the prosumers. Therefore, a control and coordination between the DSO and prosumers is key for this prominent issue, to ensure the voltage levels of the grid and while fully harnessing the most economical benefits for the different stakeholders.

The voltage of a grid-connected microgrid (in our case the low voltage (LV) portion of a distribution grid) can be optimized by relying on many different control schemes based on the communication infrastructure [2]. The conventional control architecture is a centralized one, with a master controller denoted as a microgrid central controller (MGCC), as illustrated in Fig. 1 [3]. In this architecture, intelligent devices (e.g., smart meters) and distributed energy resources (DERs) send necessary information to the MGCC, which then computes the optimal set-points for the DERs. References [4] and [5] have proposed a VAR optimization control strategy for PV systems that can be solved by a centralized control. However, a fully centralized control presents the major drawback of single point of failure, meaning that the loss of controller will endanger the overall control function of the considered microgrid. Moreover, there is lack of scalability as well as potential privacy issues leading to an unwillingness of the consumers to share their data and accept that their resources would be controlled by a third party [6].

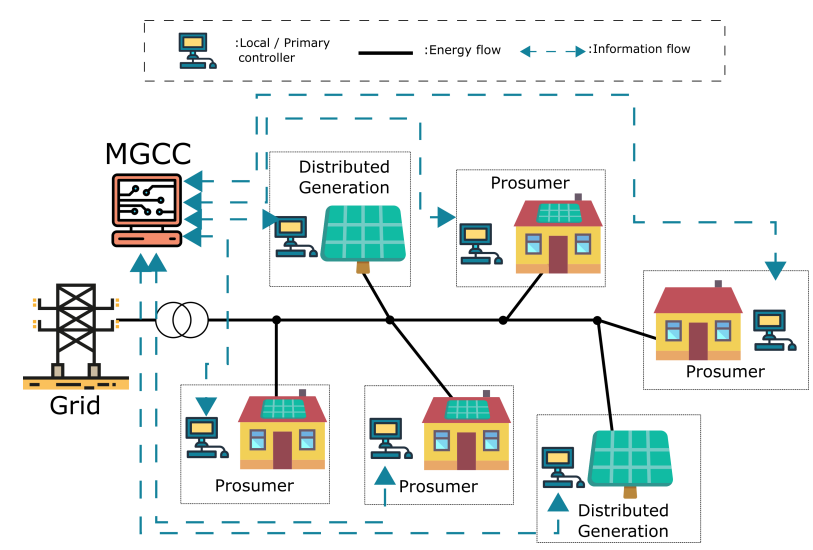

Fig. 1. Illustration of a microgrid centralized control architecture.

A distributed control architecture offers more robust control scheme. In this framework, the DERs and/or prosumers coordinate together to control the global state of the considered microgrid. The communication between prosumers and/or 
DERs are limited within neighboring area as illustrated in Fig. 2. A MGCC may still exists in a distributed architecture for a complementary function and may act as a communication interface between the considered microgrid and the DSO. To that purpose, dual-decomposition algorithm can be used to optimize active and reactive power set-points of DERs [7]. An alternating direction method of multipliers (ADMM) with linear DistFlow model is used in [8] to control reactive power of PVs. However, the accuracy of this method cannot be guaranteed since linear DistFlow model is not reflecting the actual non-linear model of the grid.

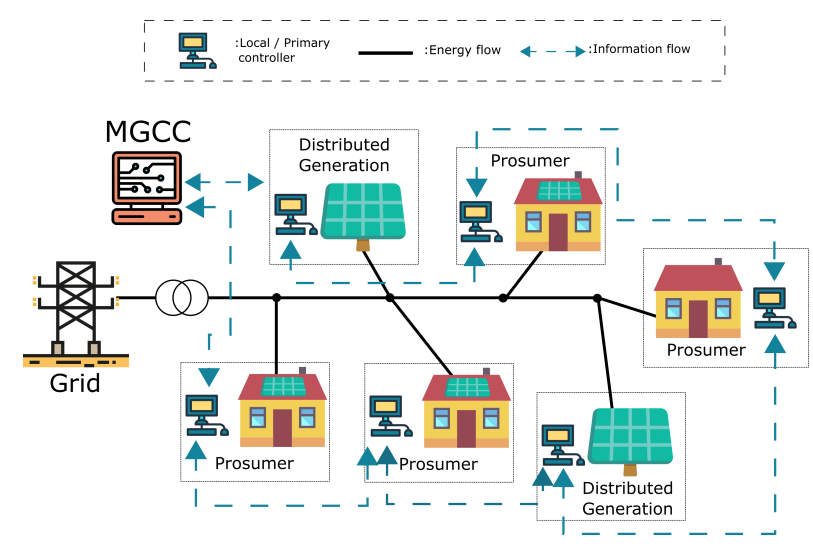

Fig. 2. Illustration of a microgrid distributed control architecture.

This paper presents a centralized and a distributed voltage controls for a radial grid-connected microgrid. We formulate the voltage control as an optimal power flow problem (OPF). Weighting parameters for line losses and PV curtailments are introduced in the OPF problem in order to have more flexibility in the controller operating mode. Solving the OPF problem ultimately returns the optimal active and reactive power set-points of all prosumers' PV according to different weighting parameters selected. The main contribution of this paper is the identification of infeasible operating points of the controllers, due to non-validity of second order cone program (SOCP) relaxation. Especially, that invalidity arises for both the centralized and distributed problem formulation. As far as the authors' knowledge, there is no study has been done that identify such infeasibilty operating points with the same control formulation as proposed in this paper.

The remainder of this paper is organized as follows. Section II describes the considered microgrid power flow formulation and PV system model. The centralized and distributed voltage control strategies are described in Section III and Section IV respectively. The controllers are simulated and compared in Section V before the conclusions are drawn in Section VI.

\section{Problem Formulation}

\section{A. Microgrid Model}

Consider a radial low voltage grid-connected microgrid with $N$ buses and represented as a graph $\mathcal{G}(\mathcal{N}, \mathcal{E})$ where $\mathcal{N}=$ $\{0,1, \ldots, N-1\}$ is the set of all buses in the microgrid and
$\mathcal{E}:=\{(i, j)\} \subset \mathcal{N} \times \mathcal{N}$ is the set of lines. For each bus $i \in \mathcal{N}$, $V_{i}$ defines the complex voltage of bus $i$. Bus 0 is the slack bus, which has a constant voltage magnitude $\left|V_{0}\right|=1$ p.u. Let $I_{i j}, P_{i j}$ and $Q_{i j}$ be the complex current, active and reactive power flowing from bus $i$ to bus $j$ respectively. The line $(i, j)$ connect bus $i$ and bus $j$ with a resistance $r_{i j}$ and a reactance $x_{i j}$.

The microgrid model lies on the conventional DistFlow equations to solve the power flow [9]. The DistFlow equations, for all links $(i, j) \in \mathcal{E}$ are expressed in (1) to (3).

$$
\begin{aligned}
P_{i j} & =P_{j b}+r_{i j} \frac{P_{i j}^{2}+Q_{i j}^{2}}{\left|V_{i}\right|^{2}}+p_{j}^{c o n}-p_{j}^{P V} \\
Q_{i j} & =Q_{j b}+x_{i j} \frac{P_{i j}^{2}+Q_{i j}^{2}}{\left|V_{i}\right|^{2}}+q_{j}^{c o n}-q_{j}^{P V} \\
\left|V_{j}\right|^{2} & =\left|V_{i}\right|^{2}-2\left(r_{i j} P_{i j}+x_{i j} Q_{i j}\right)+\left(r_{i j}^{2}+x_{i j}^{2}\right) \frac{P_{i j}^{2}+Q_{i j}^{2}}{\left|V_{i}\right|^{2}}
\end{aligned}
$$

With $b:(j, b) \in \mathcal{E}$ is the downstream neighbor of bus $j, p_{j}^{c o n}$ and $q_{j}^{c o n}$ the active and reactive power consumption of bus $j$, and $p_{j}^{P V}$ and $q_{j}^{P V}$ the active and reactive power generated by the PV at bus $j$ respectively. If there is no PV at bus $j, p_{j}^{P V}$ and $q_{j}^{P V}$ are obviously set to zero. The current for all links $(i, j) \in \mathcal{E}$ respects (4).

$$
\left|I_{i j}\right|^{2}=\frac{P_{i j}^{2}+Q_{i j}^{2}}{\left|V_{i}\right|^{2}}
$$

\section{B. PV System Model}

The subset $G:=\{g\} \subset \mathcal{N}$ denotes all the buses with connected PV. Let $\overline{p_{g}^{P V}}$ be the maximum available active power production of the PV at prosumer $g$ based on weather date (i.e., solar irradiation and temperature at the first order). The PV system (i.e., solar panel and inverter) operation is described in a $(P, Q)$ area [10] and dispatched based on the apparent power limit rating of the inverter.

$$
\begin{aligned}
\forall g \in G: & 0 \leq p_{g}^{P V} \leq \overline{p_{g}^{P V}} \\
& \left|q_{g}^{P V}\right| \leq \sqrt{\left(s_{g}^{P V}\right)^{2}-\left(p_{g}^{P V}\right)^{2}} \\
& p f_{g} \leq \frac{p_{g}^{P V}}{\sqrt{\left(p_{g}^{P V}\right)^{2}+\left(q_{g}^{P V}\right)^{2}}}
\end{aligned}
$$

Where $s_{g}^{P V}$ is the inverter apparent power and $p f_{g}$ is the predefined power factor parameter of the PV inverter $g$. In the case study, $p f$ is set to 0.95 for all PVs.

\section{Centralized Voltage Control Formulation}

The centralized voltage control is formulated as an OPF problem. Solving the proposed OPF problem provides the optimal active and reactive power set-points of all PV inverters that fulfill the voltage constraints. The proposed OPF problem is formulated in the following subsections. 


\section{A. Objective Function}

The objective of the controller is to minimize the total line losses of the microgrid and the PV curtailments. Therefore, the proposed objective functions consist of the total line losses and the PV curtailment costs expressed in (8) and (9) respectively.

$$
\begin{aligned}
\text { Loss }_{\text {tot }}^{\text {line }} & =\sum_{(i, j) \in \mathcal{E}} r_{i j}\left|I_{i j}\right|^{2} \\
\text { Cost }_{P V} & =\sum_{g \in G} C_{P V}\left(\alpha \Delta p_{g}+(1-\alpha) \Delta q_{g}\right)
\end{aligned}
$$

Where $\Delta p_{g}=\overline{p_{g}^{P V}}-p_{g}^{P V}$ and $\Delta q_{g}=q_{g, \text { init }}^{P V}-q_{g}^{P V}$, with $q_{g, i n i t}^{P V}$ the initial reactive power production of the PV $g$. It is assumed that initially $\mathrm{PV}$ owners does not require to produce/consume any reactive power (i.e., $q_{\text {, init }}^{P V}=0$ ). $C_{P V}$ represents the weight parameters for the PV curtailment that penalizes any change in the active and reactive power production of the PV. An additional weight factor $\alpha \in[0,1]$ decides the priorities of the PV control active curtailment over the reactive power deviation. Curtailing active power of the PV leads to more economic loss for the PV owner. Therefore, it may be encouraged to set the value of $\alpha$ close to one to give more weight to active power curtailment.

\section{B. Constraints}

In addition to the DistFlow equations (1) to (4) and the PV active and reactive power limit (5) to (7), the voltage limit is also defined as a constraint. This is the primary purpose of the voltage control, set to maintain the voltage of all buses within the acceptable limits.

$$
\forall i \in \mathcal{N}: \quad \underline{V_{i}} \leq V_{i} \leq \overline{V_{i}}
$$

\section{Overall Optimization Problem}

The overall OPF problem is formulated in (11), subject to (1) to (7) and (10).

$$
\begin{aligned}
\min C_{\text {loss }} \sum_{(i, j) \in \mathcal{E}} r_{i j}\left|I_{i j}\right|^{2}+ & \\
& \sum_{g \in G} C_{P V}\left(\alpha \Delta p_{g}+(1-\alpha) \Delta q_{g}\right)
\end{aligned}
$$

Where $C_{\text {loss }}$ represents the weight parameter for the grid losses. It allows seting a tradeoff between the grid losses and the curtailment of the PVs in the objective.

The equality relation of (4) is nonlinear, thus the proposed OPF problem is a non-convex.

\section{SOCP Relaxation}

To help solving the proposed OPF problem, a SOCP relaxation is used to transform the original non-convex problem into a convex problem [5]. The first of the relaxation is conducted through the introduction of two variables:

$$
\begin{aligned}
& \forall i \in \mathcal{N}: \quad v_{i}:=\left|V_{i}\right|^{2} \\
& \forall(i, j) \in \mathcal{E}: \quad \ell_{i j}:=\left|I_{i j}\right|^{2}=\frac{P_{i j}^{2}+Q_{i j}^{2}}{\left|V_{i}\right|^{2}}
\end{aligned}
$$

Next, for all links $(i, j) \in \mathcal{E}$, the nonlinear relation of (4) is relaxed into (14).

$$
\ell_{i j} \geq \frac{P_{i j}^{2}+Q_{i j}^{2}}{v_{i}}
$$

With the introduction of (12) and (13) and the current equality relaxation in (14), the OPF problem becomes convex and thus easy to solve. The detail proof of this relaxation is justified in [4], and shows that the relaxation is valid since the objective function is increased with $\ell_{i j}$. However, we identify that this relaxation is not always valid with certain pre-selected parameters, this issue will be discussed further in the section $\mathrm{V}$.

The overall relaxed OPF problem in its SOCP form can be expressed as:

$$
\begin{aligned}
\min C_{\text {loss }} \sum_{(i, j) \in \mathcal{E}} r_{i j} \ell_{i j}+ & \\
& \sum_{g \in G} C_{P V}\left(\alpha \Delta p_{g}+(1-\alpha) \Delta q_{g}\right)
\end{aligned}
$$

Subject to the:

1) DistFlow constraints: $\forall(i, j) \in \mathcal{E}$ :

$$
\begin{aligned}
& P_{i j}=P_{j b}+r_{i j} \ell_{i j}+p_{j}^{c o n}-p_{j}^{P V} \\
& Q_{i j}=Q_{j b}+x_{i j} \ell_{i j}+q_{j}^{c o n}-q_{j}^{P V} \\
& v_{j}=v_{i}-2\left(r_{i j} P_{i j}+x_{i j} Q_{i j}\right)+\left(r_{i j}^{2}+x_{i j}^{2}\right) \ell_{i j}
\end{aligned}
$$

2) Voltage constraints:

$$
\forall i \in \mathcal{N}: \quad \underline{V_{i}^{2}} \leq v_{i} \leq \overline{V_{i}^{2}}
$$

3) Current relaxtion:

$$
\forall(i, j) \in \mathcal{E}: \quad \ell_{i j} \geq \frac{P_{i j}^{2}+Q_{i j}^{2}}{v_{i}}
$$

4) PV active and reactive power limit of (5) to (7).

\section{Distributed Voltage Control Formulation}

The main objective of the distributed voltage control is to enable each prosumer to compute locally the required change of its PV active and reactive power set-point in order to maintain the global microgrid voltage within limits. A consensus ADMM is selected to optimize the microgrid voltage in a distributed fashion, due to its fast convergence speed with acceptable accuracy [11]. ADMM allows to decompose a centralized OPF (15) into a distributed problem, which relies on local decisions made at the prosumers levels.

There are two types of variables in the ADMM algorithm: local and consensus ones. Local variables are known only by individual prosumers (e.g., their voltage, incoming power flow, etc.). They are obtained by local measurement or computation. Different prosumers obviously have different local variables. Consensus variables can be interpreted as global variables that may be accessed by different prosumers. Those values are obtained from communication and coordination between a prosumer and its neighbors and are updated at each iteration of the ADMM procedure. In this paper, it is assumed that each 
prosumer is only able to communicate with its immediate bus (i.e., prosumer $j$ can only communicate with its immediate upstream bus $i:(i, j) \in \mathcal{E}$ and downstream bus $b:(j, b) \in \mathcal{E})$.

The consensus variables are used as the reference values for the local variables in the first step of the ADMM (i.e., local optimization step). Let $k$ be the iteration number of the ADMM. For each prosumer $j$, one iteration of ADMM consists of three iteration steps. In the first step, each prosumer solves the augmented Lagrangian form of the OPF problem (15) as expressed in (21).

$$
\begin{aligned}
& \min \mathcal{L}_{j}=C_{\text {loss }}\left(r_{i j} \ell_{i j}^{(j)}+\boldsymbol{\lambda}_{\boldsymbol{j}}\left(\boldsymbol{x}_{\boldsymbol{j}}-\boldsymbol{z}_{\boldsymbol{j}}\right)^{\top}+\right. \\
&\left.\frac{\rho}{2}\left\|\boldsymbol{x}_{\boldsymbol{j}}-\boldsymbol{z}_{\boldsymbol{j}}\right\|^{2}\right)+C_{P V}\left(\alpha \Delta p_{j}+(1-\alpha) \Delta q_{j}\right)
\end{aligned}
$$

Subject to PV limit constraints (5) to (7) and (22) to (26).

$$
\begin{aligned}
& P_{i j}^{(j)}=P_{j b}^{(j)}+r_{i j} \ell_{i j}^{(j)}+p_{j}^{c o n}-p_{j}^{P V} \\
& Q_{i j}^{(j)}=Q_{j b}^{(j)}+x_{i j} \ell_{i j}^{(j)}+q_{j}^{c o n}-q_{j}^{P V} \\
& v_{j}^{(j)}=v_{i}^{(j)}-2\left(r_{i j} P_{i j}^{(j)}+x_{i j} Q_{i j}^{(j)}\right)+\left(r_{i j}^{2}+x_{i j}^{2}\right) \ell_{i j}^{(j)} \\
& \ell_{i j}^{(j)} \geq \frac{\left(P_{i j}^{(j)}\right)^{2}+\left(Q_{i j}^{(j)}\right)^{2}}{v_{i}^{(j)}} \\
& \forall i \in\{i, j\}: \quad \underline{V_{i}^{2}} \leq v_{i}^{(j)} \leq \overline{V_{i}^{2}}
\end{aligned}
$$

Where $\rho$ denotes the penalty parameter of the ADMM applied to the difference between local and consensus variables. In this study, the ADMM penalty parameter is set to $\rho=2$. The vector of local variables of the bus $j$ is $\boldsymbol{x}_{\boldsymbol{j}}=\left(v_{i}^{(j)}, v_{j}^{(j)}, P_{i j}^{(j)}, P_{j b}^{(j)}, Q_{i j}^{(j)}, Q_{j b}^{(j)}\right)$, and $\boldsymbol{z}_{j}=\left(v_{i}, v_{j}, P_{i j}, P_{j b}, Q_{i j}, Q_{j b}\right)$ is the subset of consensus variables considered by the bus $j$, while $\boldsymbol{\lambda}_{\boldsymbol{j}}=$ $\left(\lambda_{v_{i}}^{(j)}, \lambda_{v_{j}}^{(j)}, \lambda_{P_{i j}}^{(j)}, \lambda_{P_{j b}}^{(j)}, \lambda_{Q_{i j}}^{(j)}, \lambda_{Q_{j b}}^{(j)}\right)$ is the Lagrange multipliers vector associated with the local variables $\boldsymbol{x}_{\boldsymbol{j}}$. The superscript $(j)$ in local variables and Lagrange multipliers means that the variables are owned and/or calculated by the prosumer $j$. The local optimization problem is a convex problem. The solution of local optimization provides the local variables for the next iteration $\boldsymbol{x}_{\boldsymbol{j}}(k+1)$.

In the second step, the prosumer $j$ communicates with its neighbors synchronously in order to update its consensus variables. Each prosumer updates the consensus variables by computing the average value of its local variables and its neighbors' local variables. The update rule for the consensus variables is (27).

$$
\boldsymbol{z}_{\boldsymbol{j}}(k+1)=\frac{1}{2}\left(\boldsymbol{x}_{\boldsymbol{j}}(k+1)+\boldsymbol{y}_{\boldsymbol{j}}(k+1)\right)
$$

Where $\boldsymbol{y}_{\boldsymbol{j}}=\left(v_{i}^{(i)}, v_{j}^{(b)}, P_{i j}^{(i)}, P_{j b}^{(b)}, Q_{i j}^{(i)}, Q_{j b}^{(b)}\right)$ is the vector of local variables received by bus $j$ from its neighbors [8].

In the last step, the Lagrange multipliers of all prosumers are updated based on the stored variables obtained from the previous ADMM steps. This update is based on the gradient ascent principles expressed in (28).

$$
\boldsymbol{\lambda}_{\boldsymbol{j}}(k+1)=\boldsymbol{\lambda}_{\boldsymbol{j}}(k)+\rho\left(\boldsymbol{x}_{\boldsymbol{j}}(k+1)-\boldsymbol{z}_{\boldsymbol{j}}(k+1)\right)
$$

\section{Simulation Results And Discussion}

In this section, the performance of the proposed centralized and distributed voltage control is evaluated on a 7-bus one phase $230 / 400 \mathrm{~V}$ feeder. Line parameters from [12] were adopted and lines' length were adjusted for the test case. In the simulations, voltage security constraints are set to $\underline{V}=$ 0.95 p.u. and $\bar{V}=1.05$ p.u.

Three PVs are located at prosumers $G=\{1,3,6\}$, with the same maximum rated power $(5 \mathrm{kVA})$ and power factor limit adjusted to $p f=0.95$. The PV profile are adopted from the irradiance data obtained from local measurments in Grenoble, France, with a time resolution of $30 \mathrm{~min}$. The load profiles are adopted from [13] with a constant power factor of 0.85 lagging, re-sampled to $30 \mathrm{~min}$.

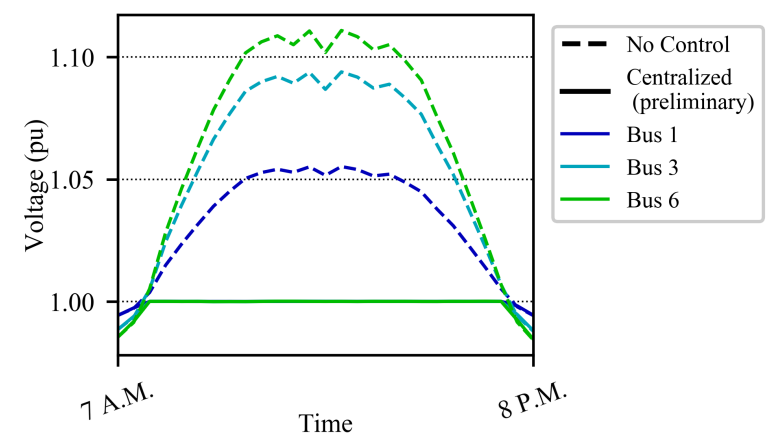

Fig. 3. Voltage magnitude profile from 7 A.M to 8 P.M. at different buses.

\section{A. Preliminary Tests of the Centralized Control}

Firstly, the centralized control is tested by using arbitrary values $C_{\text {loss }}=25, C_{P V}=1$ and $\alpha=1$. This setting is called "preliminary" throughout the paper. Fig. 3 shows the voltage profile of Bus 1, 3, and 6 from 7 A.M. to 8 P.M. with and without voltage control. Without voltage control, all buses experience over-voltages due to significant power injection. The centralized control with the preliminary settings can keep the voltage below the maximum limit. However, the controller over-regulates the voltage, as all buses are being regulated close to $1 \mathrm{p} . \mathrm{u}$, instead of remaining closer to the upper limit. Implicitly, there are two objective functions in (11). It suggests that the preliminary parameters setting tends to prioritize the loss minimization rather than the minimization of PV curtailment. Indeed, with lower PV injection, the line flows decreases, which ultimately incurs less losses.

\section{B. Sensitivity of the Centralized Control to the Weight Param- eters}

A first sensitivity analysis investigates the impact of the selection of $C_{\text {loss }}$ and $C_{P V}$ on the performance of the centralized controller in the worst hour of the day, which corresponds to the single time step when the highest overvoltages occur. Fig. 4 displays the results obtained with combinations of $C_{l o s s}$ within the range of $1 \leq C_{\text {loss }} \leq 100$, and $C_{P V}$ within the range of $1 \leq C_{P V} \leq 100$, with $\alpha=1$. 


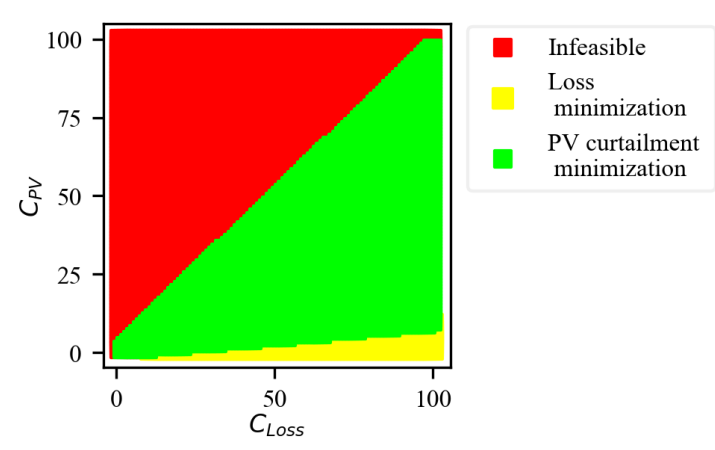

Fig. 4. Centralized controller operating regions.

Three controller operating regions are identified:

1) Region 1 (Infeasible): In this area, the branch current obtained from the relaxation (14) is significantly greater than the mathematically expected value of (4). The problem is mathematically feasible but does not respect the physics. The controller will be in the infeasible region if (29) is true (with $\epsilon_{\ell}=10^{-4}$ ).

$$
\forall(i, j) \in \mathcal{E}: \quad \ell_{i j}-\frac{P_{i j}^{2}+Q_{i j}^{2}}{\left|V_{i}\right|^{2}} \geq \epsilon_{\ell}
$$

If the condition (29) is true, then the SOCP relaxation (14) is not satisfying the equality relation of (4), which make the SOCP relaxation non-valid. This situation occurs when the losses term (8) is significantly lower than the PV curtailment term (9), thus even any significant increase of $\ell_{i j}$ will not be penalized enough. As a result, the voltage control does not work properly.

2) Region 2 (loss minimization): The control tends to minimize the line losses. It may still curtail PV production to minimize the losses even though there is no voltage violation. The preliminary setting in the previous subsection lies in this region. The controller will operate in this region if (30) is true for the single time step considered.

$$
\forall i \in \mathcal{N}: \quad V_{i}<\overline{V_{i}}
$$

3) Region 3 (PV curtailment minimization): The controller will prioritize PV curtailment minimization, thus it may curtail the PV at least as possible while maintaining the grid voltage close to the maximum voltage limit.

\section{Sensitivity of the Centralized Control to $\alpha$}

A second sensitivity analysis focuses on the operation in Region 3 with various value of $\alpha \geq 0.5$. Three values of $\alpha=[1,0.75,0.5]$ are considered. Fig. 5 shows the total PV energy curtailed for different values of $C_{\text {loss }}, C_{P V}$ and $\alpha$. We use a scatter plot to visualize the results, the greener the color, the least PV will be curtailed. In general, lower values of $\alpha$ generates a wider operating region. However, the lower the $\alpha$, the narrower the area where the PV is the least curtailed.

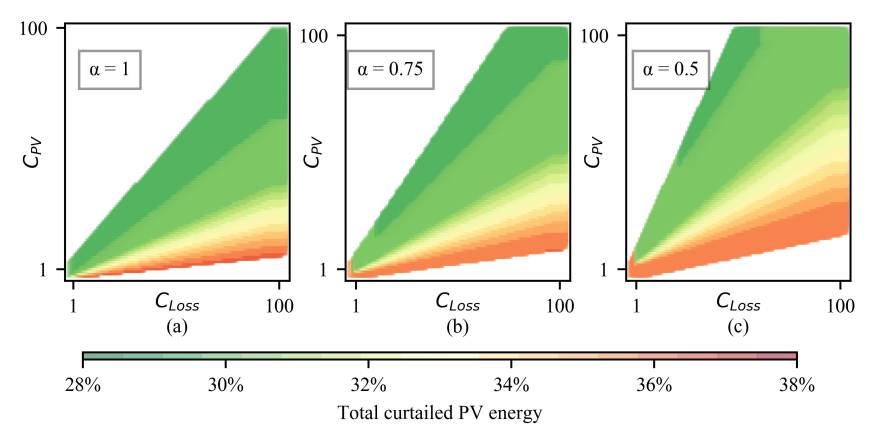

Fig. 5. The centralized controller operated in Region 3. (a) $\alpha=1$. (b) $\alpha=0.75$. (c) $\alpha=0.5$.

\section{Centralized Control Performance Test}

A last test is performed while comparing the performance of the preliminary run with a "sensitivity run" obtained with the selected parameters $C_{\text {loss }}=85, C_{P V}=80$ and $\alpha=1$.

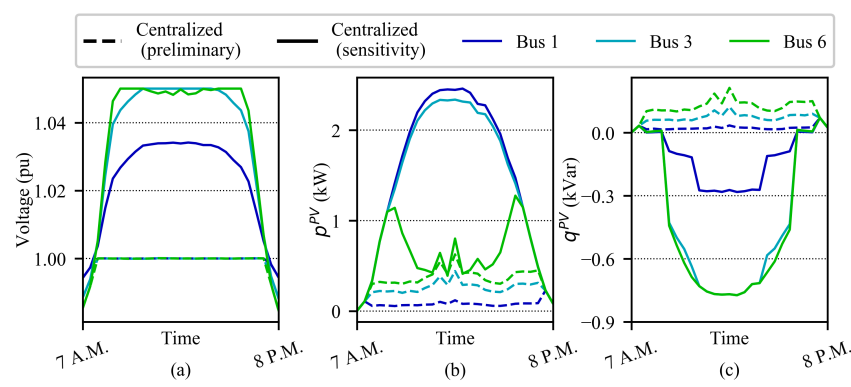

Fig. 6. Comparison between preliminary parameters and sensitivity parameters. (a) Voltage profile. (b) PV active power profile. (c) PV reactive power profile.

Fig. 6(a) shows the voltage profile with sensitivity parameters that are able to keep the voltage closer to the maximum voltage limit compared to the preliminary test. Fig. 6(b) illustrates that the preliminary parameters (Region 2) curtails more active power compared to the selected sensitivity parameters (Region 3). Moreover, the preliminary parameters produce reactive power even during the voltage rise while the selected sensitivity parameters absorb reactive power during the voltage rise, which is a more realistic behavior.

\section{E. Distributed Control Performance Test}

To test the performance of the distributed control (i.e., ADMM), we conduct the same sensitivity analysis as the centralized control to obtain the parameters that operate in Region 3 (i.e. PV curtailment minimization). Fig. 7 shows the operating Region 3 of the ADMM with different combinations of $C_{\text {loss }}, C_{P V}$ and $\alpha\left(1 \leq C_{\text {loss }} \leq 10,1 \leq C_{P V} \leq 10\right.$ and $\alpha=[1,0.75,0.5])$. A smaller interval of $C_{\text {loss }}$ and $C_{P V}$ is considered due to the increased computational time (700 s until convergence while centralized control solved in $0.5 \mathrm{~s}$ ). The parameters $C_{\text {loss }}=9, C_{P V}=6$ and $\alpha=1$ are selected based on Fig. 7. 


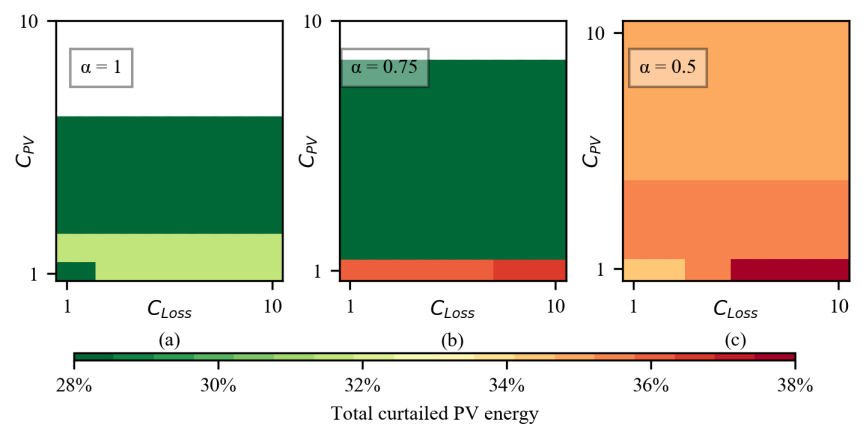

Fig. 7. The distributed controller operated in Region 3. (a) $\alpha=1$. (b) $\alpha=0.75$. (c) $\alpha=0.5$.

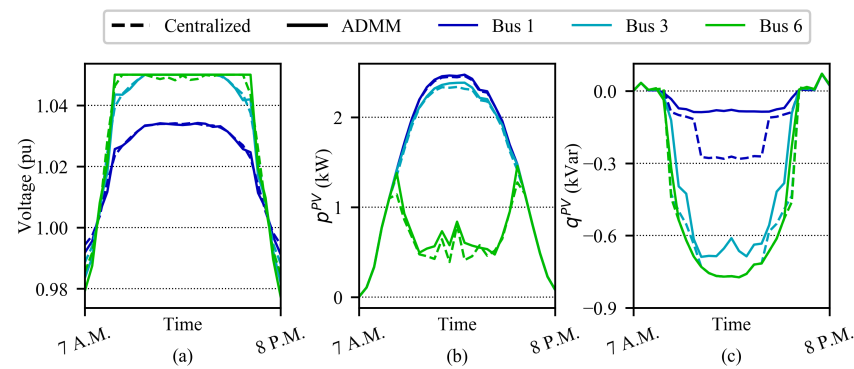

Fig. 8. Comparison between the centralized and distributed control. (a) Voltage profile. (b) PV active power profile. (c) PV reactive power profile.

Fig. 8(a) shows the voltage profile comparison between the ADMM and the centralized control with the selected parameters. It shows that both ADMM and centralized controls generate similar voltage profiles, close to the maximum limits. Similarly, Fig. 8(b) shows both the ADMM and the centralized control curtail similar profile of PV active power, despite slight differences of reactive power absorption. These comparisons show that both the ADMM and the centralized controls can effectively optimize the global microgrid voltage as long as the parameters $C_{l o s s}, C_{P V}$ and $\alpha$ for both controllers are properly defined. Furthermore, the major challenge of ADMM is the convergence rate, which strongly depends on the parameters selection. One solution is to use a decentralized controller, which is a hybridization between the distributed and centralized architecture. This represents the immediate next step of the proposed research.

\section{CONCLusion}

This paper presents a centralized and distributed voltage control strategies with ADMM for a radial LV grid-connected microgrid with a high share of local PV production. The formulation of both control strategies is based on an optimal power flow problem using a SOCP relaxation with the objective to minimize the line losses along with the curtailment of prosumers' PV subject to voltage operating limits.

The sensitivity analysis of the different weighting parameters identified three operating regions of both controllers. Especially, it showed that the convex relaxation may not be valid with some parameters selection. Therefore, the weighting parameters shall be appropriately defined in order to ensure the robustness of both controllers. Finally, the simulations results showed that both the centralized and the distributed controllers can maintain the voltage effectively.

Future works include developing a decentralized voltage control, which is an intermediate architecture between the centralized and distributed ones and to implementing economic criteria in the algorithm along with flexibilities such as storage systems.

\section{REFERENCES}

[1] M. Farrokhabadi et al., "Microgrid stability definitions, analysis, and examples," IEEE Transactions on Power Systems, vol. 35, no. 1, pp. 13-29, 2020.

[2] K. E. Antoniadou-Plytaria, I. N. Kouveliotis-Lysikatos, P. S. Georgilakis, and N. D. Hatziargyriou, "Distributed and decentralized voltage control of smart distribution networks: models, methods, and future research," IEEE Transactions on Smart Grid, vol. 8, no. 6, pp. 2999-3008, 2017.

[3] S. Sen and V. Kumar, "Microgrid control: A comprehensive survey," Annual Reviews in Control, vol. 45, pp. 118-151, 2018.

[4] M. Farivar, C. R. Clarke, S. H. Low, and K. M. Chandy, "Inverter VAR control for distribution systems with renewables," in IEEE International Conference on Smart Grid Communications (SmartGridComm), 2011, pp. 457-462.

[5] M. Farivar, R. Neal, C. Clarke, and S. Low, "Optimal inverter VAR control in distribution systems with high PV penetration," in IEEE Power and Energy Society General Meeting, 2012, pp. 1-7.

[6] H. Almasalma, J. Engels, and G. Deconinck, "Peer-to-peer control of microgrids," in IEEE Benelux PELS/PES/IAS Young Researchers Symposium, Eindhoven, Netherlands, 2016, pp. 1-6. [Online]. Available: arXiv: 1711.04070

[7] — "Dual-decomposition-based peer-to-peer voltage control for distribution networks," in CIRED - Open Access Proceedings Journal, vol. 2017, no. 1, 2017, pp. 1718-1721.

[8] P. Šulc, S. Backhaus, and M. Chertkov, "Optimal distributed control of reactive power via the alternating direction method of multipliers," IEEE Transactions on Energy Conversion, vol. 29, no. 4, pp. 968-977, 2014.

[9] M. Baran and F. F. Wu, "Optimal sizing of capacitors placed on a radial distribution system,' IEEE Transactions on Power Delivery, vol. 4, no. 1 , pp. 735-743, 1989.

[10] E. Dall'Anese, S. V. Dhople, B. B. Johnson, and G. B. Giannakis, "Decentralized optimal dispatch of photovoltaic inverters in residential distribution systems," IEEE Transactions on Energy Conversion, vol. 29, no. 4, pp. 957-967, 2014

[11] S. Boyd, N. Parikh, and E. Chu, Distributed optimization and statistical learning via the alternating direction method of multipliers. Now Publishers Inc, 2011.

[12] J. Tant, F. Geth, D. Six, P. Tant, and J. Driesen, "Multiobjective battery storage to improve PV integration in residential distribution grids," IEEE Transactions on Sustainable Energy, vol. 4, no. 1, pp. 182-191, 2012.

[13] D. Murray, L. Stankovic, and V. Stankovic, "An electrical load measurements dataset of United Kingdom households from a two-year longitudinal study," Scientific Data, vol. 4, no. 1, pp. 1-12, 2017, nature Publishing Group. 